\title{
Miscellaneous Applications
}




\title{
Speckle Noise Filtering in SAR Images Using Wavelets
}

\author{
L.Böröczky ${ }^{1}$, R.Fioravanti ${ }^{1}$, S.Fioravanti ${ }^{2}$ and D.D.Giusto ${ }^{3}$ \\ ${ }^{1}$ MSZKI-KFKI, Hungarian Academy of Sciences, Budapest, Hungary \\ boroczky@ecse.rpi.edu \\ ${ }^{2}$ Saclant Undersea Research Center, La Spezia, Italy \\ steve@saclantc.nato.int \\ ${ }^{3}$ Dept. of Electrical and Electronic Engineering, University of Cagliari, Italy \\ myrddin@elettro1.unica.it
}

\begin{abstract}
This paper presents a novel multiresolution wavelet-based algorithm for filtering SAR images in order to remove speckle noise. The basic idea is to apply to the wavelet coefficients a size-decreasing halfinterpolated median filter. The size of the median filter is adapted to the noise energy reduction between the image pyramid levels and different filter shapes are used in each wavelet subbands according to the dominant frequencies. Experimental results showed, that the proposed algorithm results in significant noise removal while the edges are preserved in the images.
\end{abstract}

\section{Introduction}

SAR technology has made possible to obtain images at fairly good resolutions when observing a ground scene from aircrafts or satellites, and it can be used to estimate also features like the dampness of the soil, the thickness of a forest, or the roughness of the sea. However, SAR images are contaminated by speckle noise, a chaotic phenomenon that results from coherent energy imaging, and that obscures the scene content and strongly reduces the possibility to observe objects.

Typical noise-smoothing methods are not well suited to preserving edge structures in speckled images; accordingly, many adaptive filters have been developed. Classical operators are based on the local variance statistics [6,4,1]. Also complex processing has been proposed; as the speckle has a different power spectrum than the signal, they can be separated by an operator in the frequency domain [3]. Some of these operator perform better in smoothing speckle in homogeneous areas, others give superior results at the vicinity of edges, but the research in this area is still open $[5,8,10]$.

Due to the inability of the Fourier theory to represent nonstationary signals, wavelet theory was developed and applied intensively also in image processing [7]. For speckle reduction of SAR images, in [9] a wavelet-based approach has been proposed, where the filtered image is obtained by a weighted linear combination of the inverse transforms and the unfiltered image.

The multiresolution method presented in this paper is also based on the wavelet 
transform, but a modified median filtering, namely a Size-Decreasing HalfInterpolated Median (SDHIM) is carried out for the wavelet coefficients. The reconstruction procedure using these filtered subbands results in smoothed SAR images with sharp edges.

In Section 2, the principles of image decomposition using wavelets are outlined. Derivation of noise energy reduction between levels of wavelet pyramid, and the SDHIM filtering of wavelet coefficients itself is described in Section 3. Experimental results obtained by the proposed algorithm using different SAR images are given in Section 4.

\section{The Wavelet Transform}

The Fourier transform, the most useful technique for frequency analysis of signals, has undesirable effects if one deals with signals which are localized in time and/or space, due to the fact that sinusoids have an infinite support.

Wavelet transform decomposes an image into basis functions, which are dilations and translations of a single prototype wavelet function:

$$
f(x)=\sum_{m=0}^{\infty} \sum_{n=0}^{\infty} c_{n}^{m} \Psi_{m, n}(x) \quad \text { where } \quad \Psi_{m, n}(x)=2^{-m / 2} \Psi\left(2^{-m} x-n\right)
$$

This transform can be implemented using band-splitting Quadrature Mirror Filters (QMF) derived from orthonormal wavelet basis.

Image decomposition by a $2 \mathrm{D}$ wavelet transform can be done by splitting an image into its low frequency part and the difference signal, which describes the difference between the lowpass image and the actual one. Due to the correlation which exists in the original image, the difference signal has a histogram that is peaked around the zero. The low frequency subband (SLL) image still contains spatial correlation. Therefore, this decomposition can be applied recursively to the lowpass band, in order to obtain a multiresolution image representation. It results in a decomposition of the signal into subbands of equal bandwidth on a logarithmic scale.

This multiresolution wavelet transform results in a compact non-redundant image representation in contrast to the traditional methods, such as lowpass filtering and Laplacian pyramid transform. The traditional pyramid-type wavelet transform recursively decomposes subsignals in the low frequency channels. However the most significant information regarding textures often appears in the middle frequency channels, at certain levels of the decomposition process [2].

At each decomposition level there are three different signals: SHL, SLH and SHH. The SHL shows scale variations in the $x$-direction and its high values indicate the presence of vertical edges. Large values of SLH and SHH indicate the presence of horizontal edges and corner points, respectively. The noise is reduced and the edges are kept if the noise-cleaning process takes into consideration both the lowpass noise reduction of SLL and the information about the edges given by SLH, SHL and SHH. 


\section{SDHIM filtering}

The SLL image of the wavelet decomposition is a lowpass image of the original one; consequently the energy of noise contained in SLL is less than the one at the previous decomposition level. The relation between the wavelet coefficients and the noise energy reduction is derived for white Gaussian noise in the following.

Let consider, by hypothesis, $x_{i}^{0}=s_{i}^{0}+n_{i}^{0}$, where $n^{0}$ is an indipendent stocastic process with $E\left\{n_{i}^{0} n_{j}^{0}\right\}=\sigma^{2} \delta_{i-j}, \delta_{i}$ being the Kronecker function.

Let $x_{i}^{l}$ being the wavelet transform coefficient obtained through the orthogonal filters $c_{i}$ such that

$$
\begin{aligned}
& \sum_{k=-\frac{N}{2}}^{\frac{N}{2}} c_{k} c_{k+2 m}=\delta_{2 m} \xi \\
& x_{i}^{l}=\sum_{h=-\frac{N}{2}}^{\frac{N}{2}} c_{h} x_{i+h}^{l-1}=\sum_{h=-\frac{N}{2}}^{\frac{N}{2}} c_{h} s_{i+h}^{l-1}+\sum_{h=-\frac{N}{2}}^{\frac{N}{2}} c_{h} n_{i+h}^{l-1}=s_{i}^{l}+n_{i}^{l}
\end{aligned}
$$

Thesis:

$$
E\left\{n_{i}^{l} n_{j}^{l}\right\}=\sigma^{2} \delta_{i-j}\left(\sum_{h} c_{h}^{2}\right)^{l}=\sigma^{2} \delta_{i-j} \xi^{l} \quad j=i+2 m
$$

Proof (by induction):

$\mathrm{E}\left\{n_{i}^{0} n_{j}^{0}\right\}=\sigma^{2} \delta_{i-j}$ by hypothesis.

If $\mathrm{E}\left\{n_{i}^{l-1} n_{j}^{l-1}\right\}=\sigma^{2} \xi^{l-1} \delta_{i-j}$ then $\mathrm{E}\left\{n_{i}^{l} n_{j}^{l}\right\}=\sigma^{2} \xi^{l} \delta_{i-j}$.

$$
\mathrm{E}\left\{n_{i}^{l} n_{j}^{l}\right\}=\mathrm{E}\left\{\sum_{\mathrm{h}=-\frac{\mathrm{N}}{2}}^{\frac{\mathrm{N}}{2}} c_{h} \cdot n_{i+h}^{l-1} \cdot \sum_{\mathrm{k}=-\frac{\mathrm{N}}{2}}^{\frac{\mathrm{N}}{2}} c_{k} \cdot n_{i+k}^{l-1}\right\}=\sum_{\mathrm{h}, \mathrm{k}=-\frac{\mathrm{N}}{2}}^{\frac{\mathrm{N}}{2}} c_{h} c_{k} \cdot \mathrm{E}\left\{n_{i+h}^{l-1} \cdot n_{i+k}^{l-1}\right\}=
$$

due to the hypothesis

$$
=\sum_{\mathbf{h}, \mathrm{k}=-\frac{\mathrm{N}}{2}}^{\frac{\mathrm{N}}{2}} c_{h} c_{k} \cdot \sigma^{2} \xi^{l-1} \delta_{i+k-(j+h)}=\sum_{\mathrm{h}, \mathbf{k}=-\frac{\mathrm{N}}{2}}^{\frac{\mathrm{N}}{2}} c_{h} c_{k} \cdot \sigma^{2} \xi^{l-1} \delta_{(k-h)-2 m}=
$$

due to the wavelet orthogonality hypothesis 
$=\sigma^{2} \xi^{l-1} \sum_{\mathrm{h}=-\frac{\mathrm{N}}{2}}^{\frac{\mathrm{N}}{2}} c_{h} c_{h+2 m}=\sigma^{2} \xi^{l-1} \delta_{i-j} \quad$ (QED).

\section{The size-decreasing half-interpolated median (SDHIM)}

The above presented derivation shows, that for logarithmic version of the images, the multiplicative noise can be considered as additive one. Furthermore, considering some assumptions, in wavelet subbands the white Gaussian nature of the noise is remained. Taking into account these facts, the SDHIM filtering is applied to the wavelet subbands that are obtained by the wavelet transform of the logarithmic image. The speckle noise energy of a certain pyramid level is decreased relative to the previous decomposition level, if the input image is the logarithmic version of the original one. Therefore, the size of median filter applied at each wavelet decomposition level has to be adapted to this relation.

The initial size of the median is decreased by a factor $\alpha=2 \lambda_{n r}$, where $\lambda_{n r}$ is the noise reduction factor depending on the wavelet coefficients. The factor 2 is due to the subsampling performed at each decomposition level. In order to preserve edges in the original image, different shapes of median filter (horizontal, vertical, diagonal) are used in each wavelet decomposed image, according to the dominant frequency in that particular subband.

To allow for the use of median filters with size inferior to $1 \times 3$ or $3 \times 1$ pixels, a linear interpolation of adjacent pixels has been performed. In the case of the interpolated median, the output of the filter is the median between the central pixel and two linearly interpolated pixels computed by the relation:

$i_{p}=c_{p}+\left(n_{p}-c_{p}\right) s_{m}$

where $i_{p}$ is the interpolated pixel, $c_{p}$ the central pixel, $n_{p}$ the nearest real pixel in the chosen direction, and $s_{m}$ is the is the half size of the median $\left(s_{m} \leq 1\right)$.

The final logarithmic image is obtained as a minimum between the original logarithmic image and the reconstructed one, in order to prevent additional noises originated by the wavelet-based filtering. The exponential function of this reconstructed image gives the final filtered image.

\section{Experimental results}

Experiments were carried out for the proposed speckle removal algorithm using different SAR images. At first, the natural logarithm was taken for each pixel value of the original image. Then, this "logarithmic" image was decomposed by a 2-level wavelet transform using filter coefficients presented in [7]. The SDHIM filtering was applied only to the highpass subbands (SLH, SHL, SHH) with the initial filter size of $1 \times 5$. For the lower resolution subband images the filter size was decreased by $\alpha=5 / 3$, and an interpolation was carried out by $s_{m}=0.5$. The exponential function 
was applied to the reconstructed logarithmic images in order to get back the conventional pixel values of the filtered image.

Two original SAR images are shown in Figure 1 and Figure 2. The filtered images resulted by the proposed speckle reduction process are shown in Figure 3 and Figure 4, respectively. In both filtered images an obvious reduction in the speckle can be seen in homogeneous regions with good edge preservations. Furthermore, the histogram of the filtered image in Figure 4 becomes bimodal in contrast to the unimodal one of the unfiltered image in Figure 2 (Figures 5 and 6).

\section{Conclusions}

A multiresolution filtering algorithm, based on wavelets and consists of a special median filtering of wavelet coefficients, was proposed for speckle removal of SAR images. This novel approach resulted in a significant smoothing in homogeneous areas while edges are preserved in the images. Theoretical investigations of speckle noise distribution in wavelet subbands and its application to the filtering method are topics of our future research.

\section{Acknowledgments}

Lilla Böröczky and Roberto Fioravanti acknowledge the European Community Phare-Accord Mobility Grant H9112-0267. Lilla Böröczky acknowledges also the support by the Hungarian National Science Research Fund F014077.

\section{References}

1. S.M.Ali, R.E.Burge: New automatic techniques for smoothing and segmenting SAR images. Signal Processing 14 (4), 333-344 (1988)

2. T.Chang, C.-C.J.Kuo: Texture analysis and classification and tree-structured wavelet transform. IEEE Trans. Image Processing 2 (4), 429-441 (1993)

3. R.A.Cordey, J.T.Macklin: Complex SAR imagery and speckle filtering for wave imaging. IEEE Trans. Geoscience and Remote Sensing 27 (6), 666-673 (1989)

4. J.M.Durand, B.J.Gimonet, J.Perbos: SAR data filtering for classification. IEEE Trans. Geoscience and Remote Sensing 25 (5), 629-637 (1987)

5. T.Hosomura, C.W.Jayasekera: Speckle filtering and texture analysis in SAR images. Proc. IEEE-IGARSS'93, 1423-1425 (1993)

6. J.S.Lee: Speckle suppression and analysis for synthetic aperture radar images. Optical Engineering 25 (5), 636-645 (1986)

7. S.G.Mallat: A theory for multiresolution signal decomposition: The wavelet representation. IEEE Trans. Pattern Analysis and Machine Intelligence 11 (7), 674-693, 1989

8. F.J.Martin, R.W.Turner: SAR speckle reduction by weighted filtering. Int. Journal of Remote Sensing 14 (9), 1759-1774 (1993)

9. M.C.Proença, J.-P.Rudant, G.Flouzat: Using wavelets to get SAR images free of speckle. Proc. IEEE-IGARSS'92, 887-889 (1992)

10.M.R.Zaman, C.R.Moloney: A comparison of adaptive filters for edge-preserving smoothing of speckle noise. Proc. IEEE-ICASSP'93, V/77-80 (1993) 


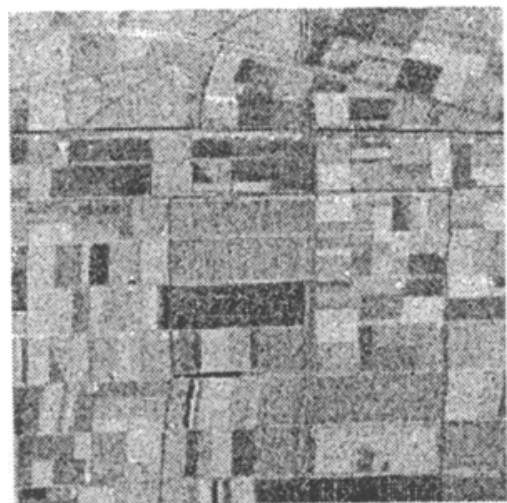

Fig. 1. Original SAR image.

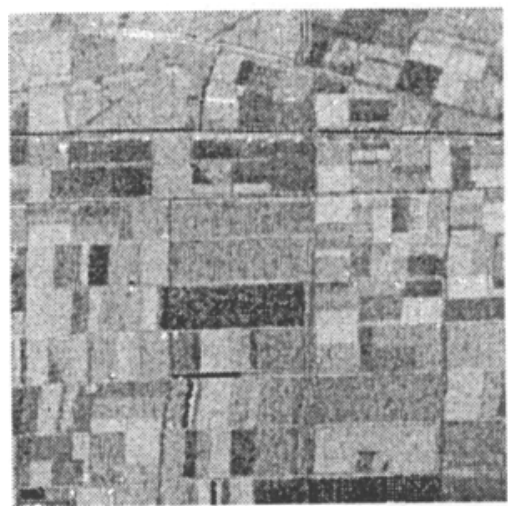

Fig. 3. Filtered SAR image.

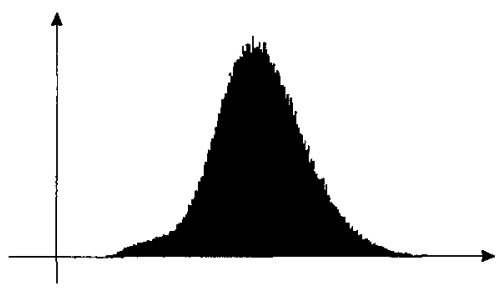

Fig. 5. Histogram of image in Fig. 2.

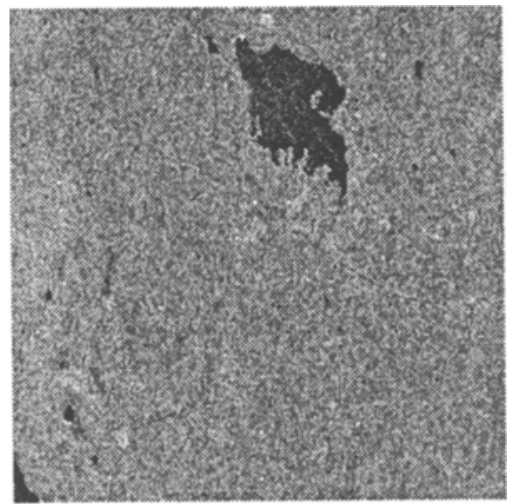

Fig. 2. Original SAR image.

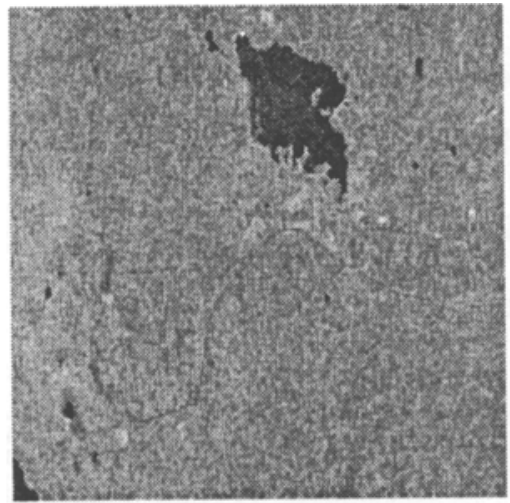

Fig. 4. Filtered SAR image.

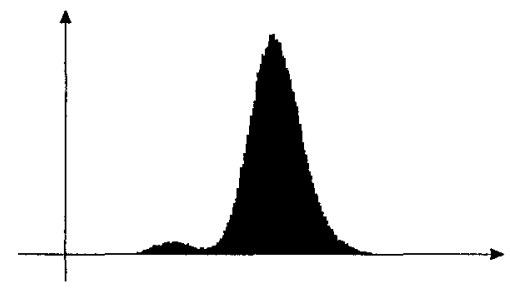

Fig. 6. Histogram of image in Fig. 4. 